\title{
The Measurement of the QT Interval
}

\author{
Pieter G. Postema* and Arthur A.M. Wilde
}

Heart Center, Department of Cardiology, Academic Medical Center, Amsterdam, The Netherlands

\begin{abstract}
The evaluation of every electrocardiogram should also include an effort to interpret the QT interval to assess the risk of malignant arrhythmias and sudden death associated with an aberrant QT interval. The QT interval is measured from the beginning of the QRS complex to the end of the T-wave, and should be corrected for heart rate to enable comparison with reference values. However, the correct determination of the QT interval, and its value, appears to be a daunting task. Although computerized analysis and interpretation of the QT interval are widely available, these might well over- or underestimate the QT interval and may thus either result in unnecessary treatment or preclude appropriate measures to be taken. This is particularly evident with difficult T-wave morphologies and technically suboptimal ECGs. Similarly, also accurate manual assessment of the QT interval appears to be difficult for many physicians worldwide. In this review we delineate the history of the measurement of the QT interval, its underlying pathophysiological mechanisms and the current standards of the measurement of the QT interval, we provide a glimpse into the future and we discuss several issues troubling accurate measurement of the QT interval. These issues include the lead choice, U-waves, determination of the end of the T-wave, different heart rate correction formulas, arrhythmias and the definition of normal and aberrant QT intervals. Furthermore, we provide recommendations that may serve as guidance to address these complexities and which support accurate assessment of the QT interval and its interpretation.
\end{abstract}

Keywords: QT interval, long QT syndrome, arrhythmias, sudden death.

\section{INTRODUCTION}

The evaluation of every electrocardiogram should also include an effort to interpret the QT interval to assess the risk of malignant arrhythmias and sudden death associated with an aberrant QT interval. The QT interval is measured from the beginning of the QRS complex to the end of the Twave, and should be corrected for heart rate to enable comparison with reference values. However, the correct determination of the QT interval, and its value, appears to be a daunting task. Although computerized analysis and interpretation of the QT interval are widely available, these might well over- or underestimate the QT interval and may thus either result in unnecessary treatment or preclude appropriate measures to be taken $[1,2]$. Similarly, also accurate manual assessment of the QT interval, even of rather straightforward ECGs, appears to be difficult for many physicians worldwide. In this review we delineate the history of the measurement of the QT interval, its underlying pathophysiological mechanisms and the current standards of the measurement of the QT interval, we provide a glimpse into the future and we will discuss several issues troubling accurate measurement of the QT interval.

\section{QT HISTORY}

To acquire an impression of the value of an aberrant QT interval, we will first discus the earliest recognitions of the QT interval and the cognizance of its importance that developed through the years.

*Address correspondence to this author at the Department of Cardiology, Academic Medical Center, P.O. box 22660, 1100 DD Amsterdam, The Netherlands; Tel: +31-20-5663072; Fax: +31-20-6971385;

E-mail: P.G.Postema@ cardiologie-amc.nl
The labeling of the human electrocardiographic waveforms was accomplished by Einthoven in 1895 [3]. At that time, he determined the P-wave, QRS-complex and the Twave. Several years later, in 1906, he also recognized a Uwave in a single patient [4], which later was actually recognized in most electrocardiograms by him and Lewis $[5,6]$.

Abnormal T-wave morphologies were considered pathological, but the value of the QT interval was not much appreciated at that time. In 1950, Wolff stated: "The Q-T interval is measured from the beginning of the QRS complex to the end of the $\mathrm{T}$ wave, but it is not often necessary to determine this value" [7]. In that time, a prolonged QT interval was predominantly recognized during the sequels of myocardial infarction.

However, it was only a few years later, in 1957, that Jervell and Lange-Nielsen described a family in which 4 out of the 6 children had a peculiar heart disease in combination with deafness [8]. No signs of structural heart disease could be found in these children, however, their electrocardiograms revealed prolongation of the QT interval in all examined cases. Moreover, these children suffered from fainting attacks, and three of the children died suddenly at the age of 4 , 5 and 9 years. The oldest, a boy, was known with fainting attacks during effort and all medical examinations for these attacks were normal except for his prolonged QT interval. Jervell and Lange-Nielsen, noted that the QT lengthened even more after stair running, subcutaneous adrenaline and quinidine despite almost similar RR intervals as compared to a resting condition. In contrast, digitalis and atropine resulted in shortening of the QT interval. However, only one week after discharge, the boy suddenly died. Macroscopic and microscopic autopsy revealed normal cardiac anatomy and 
no other diseases. His sister, only 5 years old, died suddenly in the same year while picking berries. She was known with previous fainting attacks during swimming and running but was never medically examined. The last sister to die was only 4 years old. She had previous fainting attacks, but suddenly died when playing with her remaining sisters. A medical examination before her death was also normal, except for her prolonged QT interval on several occasions. Of interest, mechanical systole evaluated by phonocardiography, was not correspondingly lengthened in these patients [9].

In the years thereafter, many more families with frequent premature sudden death and prolonged QT intervals, more often without associated deafness, were recognized. For example, it was only one year after the description of Jervell and Lange-Nielsen, in 1958, that a presumably healthy boy with a prolonged QT interval was uncovered (Fig. 1) who had a family history of sudden death and many years later appeared to have a type-3 Long QT syndrome. This boy even belongs to one of the largest and best characterized families worldwide [10].

In 1963, Romano, Gemme and Pongiglione described a 3-month old child with normal hearing, a prolonged QT interval, frequent syncopes and 'paroxysms' of ventricular fibrillation during attacks. Two brothers of the child with identical clinical symptoms, had died at the age of 44 days and 4 months [11].

In the next year, 1964, Ward described a girl who at the age of 16 months already had frequent fainting attacks, especially during play [12]. After frequent examinations a psychological basis was suspected and she was finally referred to a children's psychiatrist. However, at the age of 6.5 years the psychiatrist referred her back for further medical examinations, as the symptoms did not impose entirely psychogenic. At that time an ECG revealed an extremely prolonged QT interval but no arrhythmias. In view of the objective observations of the parents, it was decided to let her run around the ward. The effect was dramatic; the child collapsed after 80 yards in ventricular fibrillation. Luckily, the child recovered and after many more attacks (most apparent during fever), treatment with pronethalol (one of the earliest non-selective beta-blockers but later abandoned due to serious side effects in mice) resulted in suppression of the attacks. Her brother, who also had a prolonged QT interval and frequent syncopes, was also treated with pronethalol, but later switched to propranolol. However, he remained to have frequent attacks. This notwithstanding, after his propranolol was withheld for one day he had a last severe attack and died.
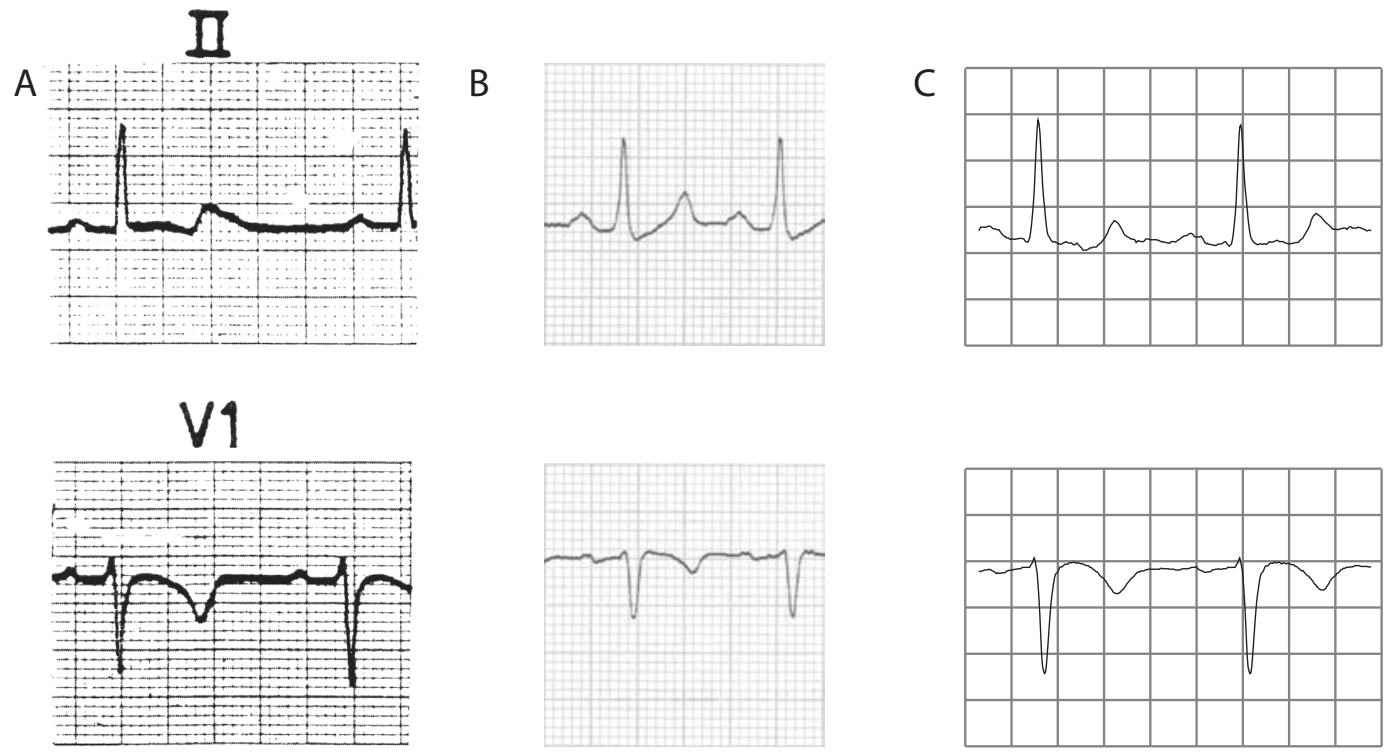

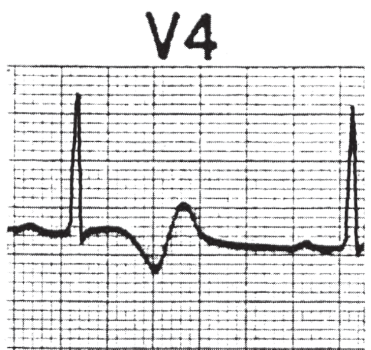

1958

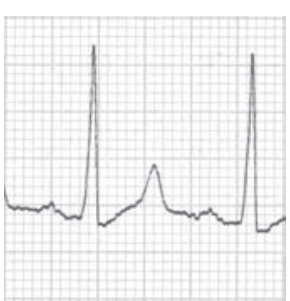

1988

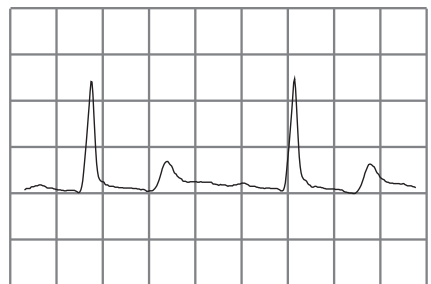

2008

Fig. (1). ECGs during 50 years of follow-up of the first family member identified from a large family with the $S C N 5 A 1795$ insD mutation. Leads II, V1 and V4 are shown with standard calibration. Note the severe repolarization abnormalities indicative of long-QT syndrome type3 already apparent on the first ECG (A). During those 50 years, the PQ and QRS interval clearly prolong, indicative of progressive cardiac conduction defects in both atria and ventricles (B and C) as has uniquely been associated with the SCN5A 1795insD mutation. Figure reproduced from Postema et al. with permission [10]. 
It lasted several decennia until the genetic underpinning of the Jervell-Lange-Nielsen syndrome (Long QT syndrome with deafness) and Romano-Ward syndrome (Long QT syndrome without deafness) was finally uncovered $[13,14]$. At present, we are familiar with potentially disease causing sequence variations in 13 genes and a similar number of (sub-)types of Long QT syndrome. Moreover, it was not until 2000 before the first descriptions of the mirror image syndrome emerged; Short QT syndrome [15]. Also this entity is related to familial sudden death due to ventricular fibrillation and at present 3 genetic types are known [16-19].

Currently, cognizant of the possible dramatic effects of a prolonged QT interval the FDA even recommends to test all new drugs for their ability to prolong the QT interval [20].

\section{QT AND CARDIAC REPOLARIZATION}

After this summary of the history of the QT interval and its directly associated pathology, we will now discuss its underlying pathophysiological mechanisms. The QT interval is a measure of the combination of cardiac depolarization and repolarization as it encompasses both the QRS complex and the J-T interval. Likewise, also ventricular conduction delay is often associated with (a lesser degree of) lengthening of the QT-interval. Cardiac depolarization is dependent of fast inward sodium current $\left(I_{\mathrm{Na}}\right)$ through the cardiac sodium channels of which the $\alpha$-subunit is encoded by the SCN5A gene (Fig. 2). Cardiac repolarization is determined by the interplay between (and among others) persisting inward sodium current $\left(I_{\mathrm{Na}}\right)$, slow inward calcium current $\left(I_{\mathrm{Ca}-}\right.$ L) and outward potassium currents $\left(I_{\mathrm{to}}, I_{\mathrm{Kr}}, I_{\mathrm{Ks}}, I_{\mathrm{K} 1}\right)$. CACNAIc encodes the $\alpha$-subunit of the calcium channel, while the four different potassium channel $\alpha$-subunits are encoded by the $K C N D 3, K C N H 2, K C N Q 1$ and $K C N J 2$ gene respectively.

When these channels are subject to a gain-of-function or a loss-of-function (e.g. due to drugs, mutations or a change in electrolyte levels), this will be mirrored in current density, subsequent modulation of the cardiac action potential and finally in changes in the duration of the QRS complex and/or the QT-interval. For example, a loss-of-function mutation in the $K C N H 2$ gene will result in less repolarizing $I_{\mathrm{Kr}}$ and subsequent delay of repolarization and will thus result in a

A

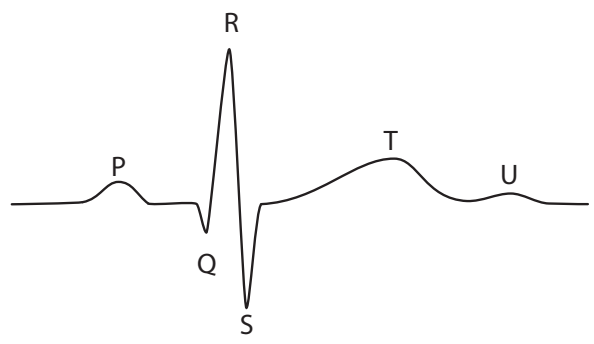

B
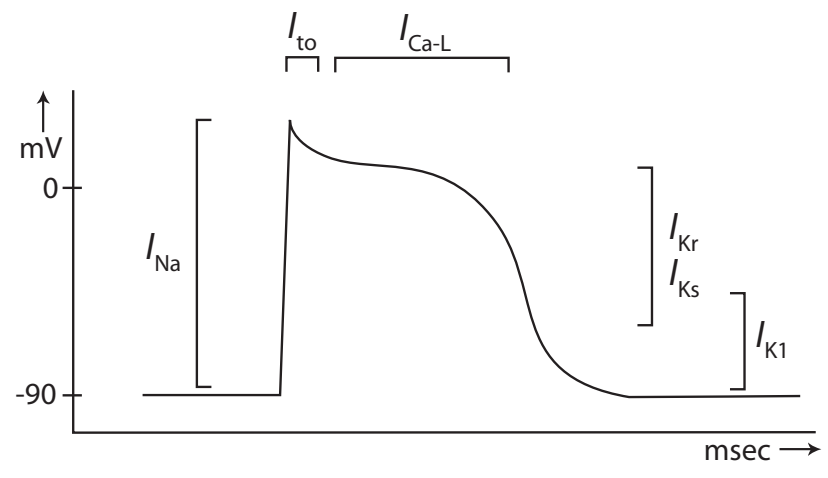

C

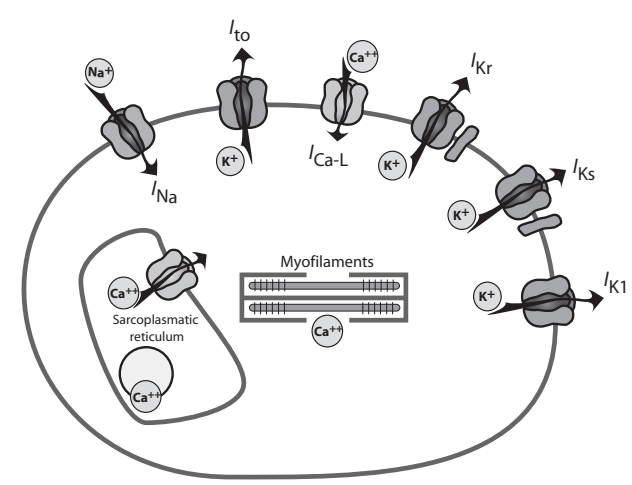

Fig. (2). Schematic representation of the ionic currents contributing to the action potential; A. the electrocardiogram (ECG) and the P-QRS-T segments in time aligned with B; B. the ventricular action potential and the ionic currents originating from C; C. the cardiomyocyte displaying only those cardiac ion currents as mentioned in the text. 
longer action potential and a prolonged QT interval. Importantly, one should realize that there are also $\beta$-subunits that modulate these ion channels and that calcium influx through calcium channels play a critical role in triggering calcium release from the sarcoplasmatic reticulum followed by contraction. It should also be noted that there remains to be a controversy on the exact mechanism by which cardiac repolarization occurs at the level of the whole heart and how the T-wave is finally inscribed on the ECG [21-24].

\section{QT PROBLEMS}

There are already several excellent reviews on the measurement of the QT interval and its associated problems. For example, Lepeschkin and Surawicz already covered these items in 1952 [25], and Garson in 1993 [26]. In this section we will provide an up-to-date summary of the papers of these and other authors. It should be noted that we here discuss the bedside measurement of the QT interval from a standard 12-lead ECG of 10 seconds. There are also more sophisticated techniques that we will discuss later. The QT interval should be measured from the beginning of the QRS complex to the end of the T-wave. Please note that Garson already commented that 'this seemingly innocuous definition is fraught with problems'.

\section{QT Problem 1: Which Lead?}

The QT interval is different in different leads (as all ECG parameters are). This is caused by the varying projections on different lead vector axes. Historically, measurement of PQRS-T intervals are preferably performed in lead II. Lewis already measured PR intervals in lead II in 1912, as did Bazett for QT intervals in 1920 [6-27]. This custom has been continued through the years, until now [28, 29]. There are several reasons for this custom; first, in the earliest years of the ECG there were no precordial leads and lead choice was thus confined to the limb leads. Furthermore, the vector axis of the P, QRS and TU-wave is predominantly directed infero-lateral and thus in the direction of lead II. This often results in easily recognizable $\mathrm{P}, \mathrm{QRS}, \mathrm{T}$ and $\mathrm{U}$-waves in lead II, which promotes their measurement. As a consequence, the reference values are also determined for lead II [28]. Furthermore, the QT interval was found to be longest in lead II in most children with Long QT syndrome [30]. Sometimes, the T-wave will not be easily measured in lead II, and alternatively lead V5, V6 or I can be used.

\section{QT Problem 2: What About U?}

The QT interval implies that the U-wave should not be included in the measurement, as one would then need to determine the QU interval. However, it is clear that cardiac repolarization is only finished after the end of the U-wave (Fig. 2). But as the U-wave is often suppressed by standard ECG filtering or suppressed by the P-wave at faster heart rates, it is often not well recognized on a standard 10 second ECG. Therefore it is common practice to measure the QT interval instead of the QU interval as a measure of the duration of cardiac repolarization. In fact, there are also no accepted reference values for the QU interval and often dedicated electrocardiographic techniques are needed before one can really focus on the U-wave [31]. In some pathologies, e.g. Andersen-Tawil syndrome (denoted by a loss-offunction of the $K C N J 2$ gene and resulting in less late rectifying $I_{\mathrm{K} 1}$ current), the U-wave will increase significantly [31, 32]. When the U-wave is meticulously studied, it has a duration of 200 to 350ms and inclusion in the QT interval would thus result in extreme and erroneous QT values [31]. Still, when it is accepted that the TU-wave is the reflection of a continuous process of repolarization, it is no surprise that defining the true end of the T-wave is complicated.

\section{QT Problem 3: Where Does QT end?}

The discrimination between the T-wave and U-wave can indeed be extremely difficult, especially in the presence of abnormal TU morphologies. But also on ECGs with normal TU morphologies this seems to be a daunting task. In 2005 Viskin and colleagues performed a study among 877 physicians worldwide and asked them to determine the QT interval on 4 rather straightforward ECGs (i.e. with concordant T-waves of sufficient amplitude during regular sinus rhythm) and to classify these QT intervals as either normal or prolonged. Their answers were compared to a gold standard as set by 25 world-renowned QT experts [33]. It appeared that less than $25 \%$ of the 771 cardiologists and non-cardiologists in this study correctly classified all 4 QT intervals as being either normal or prolonged. The authors thus concluded that 'the majority of physicians cannot recognize a long QT when they see one' [33]. Probably there are three main issues why these physicians erred; 1) the definition of the end of the Twave, 2) the correction for heart rate and 3) cut-off values.

In 2008 we performed a proof-of-principle study with the aim to show that a simple guideline on how to measure the QT interval helps in this respect [34]. Lepeschkin and Surawicz already advocated the first step in this guideline in 1952, i.e. the definition of the end of the T-wave, also known as the tangent method. They used a tangent drawn to the steepest last limb of the presumed T-wave to define the end of the T-wave as the intersection of this tangent with the baseline (i.e. the U-P segment) [25]. Some authors, including us, use this method on a day-to-day basis on normal and abnormal TU morphologies (Fig. 3). Second, and important, the acquired QT interval should be corrected for heart rate, i.e. the QTc interval, to enable comparison with reference levels (see also next paragraph). In our proof-of-principle study, we learned medical students how to discriminate normal and abnormal QT intervals with this method in conjunction with Bazett's correction formula $\left(\mathrm{QTc}=\mathrm{QT} / \mathrm{RR}^{1 / 2}\right.$ [sec]) and a simple cut off value of $450 \mathrm{~ms}$, on the same ECGs as used by Viskin and colleagues, and they performed extremely well [34]. In contrast, other authors including world-renowned QT experts have advocated the use of the $\mathrm{TU}$ nadir (i.e. the point between the $\mathrm{T}$ and $\mathrm{U}$ wave which is closest to the baseline) to determine the end of the T-wave [35]. This seems to result in slightly longer QT intervals [36], but further studies are underway.

It should be noted again that the determination of the true end of the T-wave is difficult, if not impossible. In the Viskin study the QTc range between world-renowned QT experts was already 34 to $80 \mathrm{~ms}$. Ideally, measurements are performed on-screen on digitalized ECGs and the average QT of 3 or more beats are determined during stable sinus 


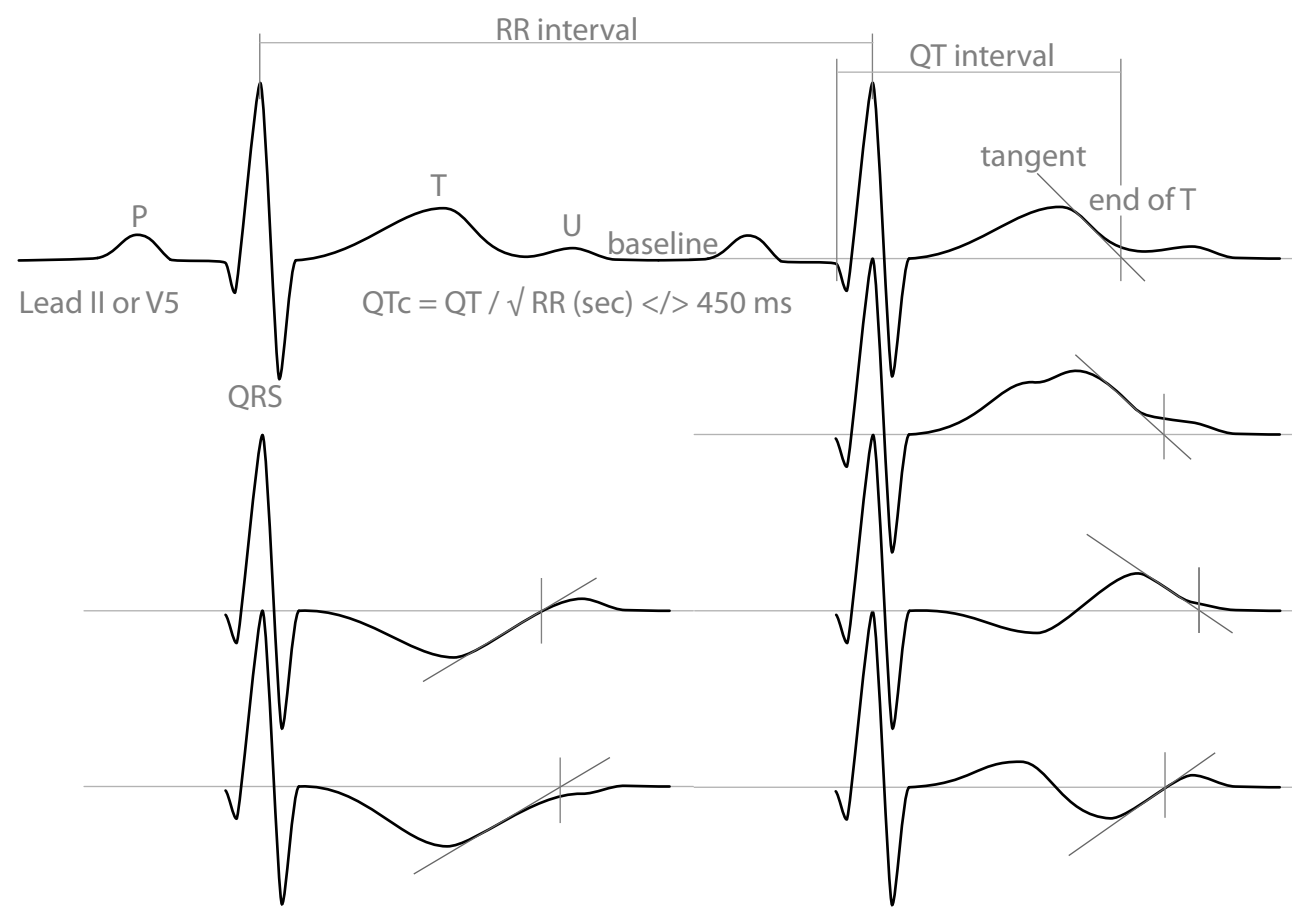

Fig. (3). Schematic illustration of the use of the tangent method to define the end of the T-wave in normal and abnormal TU morphologies, which then promotes the determination of the QTc interval.

rhythm to decrease measurement variation. Future studies will shed a light on the differences that are introduced by using different guidelines to measure the QT. Notably, when the T-wave is notched or bifid, and the U-wave not easily recognized, it will become even more complicated to define the end of T. In practice, one should aim at the largest wave in the TU-complex and measure the end of that wave (Fig. 3).

\section{QT Problem 4: Correction for Heart Rate}

Normal cardiac repolarization adapts to heart rate. This critical feature ensures that with increasing heart rate, the myocardium remains constantly excitable, i.e. completely repolarized, before the next depolarization wave enters. This prevents incomplete repolarization and the subsequent possibility for re-entrant tachycardia. In Long QT syndrome, and especially in $I_{\mathrm{Ks}}$ related Long QT syndrome (type 1 ), cardiac adaptation to changes in heart rate is disrupted, which promotes arrhythmias. However, QT correction for heart rate is imperfect and full of controversies.

One of the earliest efforts to acquire a standardized heart rate correction formula was made by Bazett in 1920 (QTc = $\mathrm{QT} / \mathrm{RR}^{1 / 2}$ [sec], where $\mathrm{RR}$ is determined in the preceding RR interval) [27]. His method has been used ever since [12, $34,35]$. This exponential method enabled the comparison of QT intervals at different heart rates. However, it works best between 60 and 100 beats per minute, while it may give erroneous results at both slower (overcorrection) and faster heart rates (undercorrection) [37]. The Fridericia exponential correction $\left(\mathrm{QTc}=\mathrm{QT} / \mathrm{RR}^{1 / 3}\right.$ [sec]) [38] has also been developed in 1920 and has the same limitations at slow heart rates, but is considered to be more accurate than Bazett's correction at faster heart rates. Another rather often used correction method is the linear Framingham method $(\mathrm{QTc}=$ $\mathrm{QT}+0.154(1-\mathrm{RR})$, which results in more uniform rate cor- rection over a wider range of heart rates. There are many more rate correction methods available, all with different shortcomings [35, 39]. A recent study by Barsheshet and colleagues from many different centers worldwide even suggested that QT correction in Long QT syndrome patients should be genotype specific to result in optimal risk stratification[40]. Still, Bazett's correction is most commonly used.

\section{QT Problem 5: Arrhythmias}

The QT interval is preferably measured during stable sinus rhythm and then even preferably determined from the average of 3 consecutive beats. However, in some occasions we are challenged with overt sinus arrhythmia, extra systoles or even atrial fibrillation. Extra systoles and the beat directly following an extra systole should not be included in the measurement of the QT interval but do provide a lot of information; those patients with decreased repolarization reserve will show aberrant QT intervals or even giant T-U waves after the sudden change in heart rate initiated by the extra systole. ${ }^{41}$ In sinus arrhythmia, especially apparent in (young) children, the varying RR intervals result in so much variation in the QTc interval that the probability for an erroneous diagnosis of a prolonged QT is increased. Subsequently, too many children may end up with a diagnosis of a prolonged QT interval. The same probably holds for atrial fibrillation in which the shortest RR intervals probably result in prolonged QTc values. Therefore, the QTc is preferably determined during stable sinus rhythm, while QTc values derived from ECGs with arrhythmias should be interpreted with caution.

\section{QT Problem 6: Normal, too long or too short?}

So what actually is a normal QT interval? Viskin wrote an excellent review on this topic in 2009 [42]. One should 
realize that there is no single cut off value that distinguishes all Long or Short QT syndrome patients from healthy controls; there will always be overlap. This is caused by the fact that there are many QT syndrome patients with in fact a normal QT interval. These patients are carriers of a pathogenic mutation in one of the QT genes, but they apparently have modifier genes that in the end result in a normal QT interval [43]. However, this does not mean that they may not have a slightly increased risk for arrhythmias or even death [44], or will respond with critical QT lengthening upon QT prolonging drugs. Furthermore, they may have severely affected children or siblings. In patients suspected of Long QT syndrome in family screening, a cut off value of $430 \mathrm{~ms}$ seems to discriminate best between those with and without Long QT syndrome [45].

When one would determine the upper and lower 2.5 percentile of the QT interval in the population as either being too long or too short, the available population studies suggest that normal QTc values for males are between 350 and 450 $\mathrm{ms}$ and for females between 360 and $460 \mathrm{~ms} .^{42}$ Still, especially those patients with extremely short or prolonged QT intervals will be at highest risk for arrhythmias. A QTc cut off value of $500 \mathrm{~ms}$ in congenital Long QT syndrome patients at which the risk for arrhythmias is significantly increased, has been suggested by Priori and colleagues [46, 47]. It seems reasonable to also use this threshold for patients with acquired Long QT syndrome. For Short QT syndrome this value would be around $330 \mathrm{~ms}$.

\section{QT MEASUREMENT TIPS AND TRICKS}

So we have discussed several problems with the measurement of the QT interval, now how to proceed? The first recommendation in the case of a possible aberrant QT interval is to repeat the ECG. Especially in the presence of difficult TU morphologies this may be very worthwhile. We are accustomed to the use of the tangent method in lead II, in combination with Bazett's formula and a QTc cut off value of $450 \mathrm{~ms}$ (Fig. 3) [34]. In acquired prolonged QT (e.g. in the case of severe hypokalemia) we use a QTc cut off value of $500 \mathrm{~ms}$ to identify those patients at highest risk for events.

There are several additional techniques to uncover decreased safety of repolarization, i.e. loss of repolarization reserve. These methods are aimed at introducing an increase and decrease in heart rate to be able to study QT adaptation and potentially loss of repolarization reserve. An elegant test is to start with a supine ECG and then let the patient stand up briskly. The brief tachycardia associated with brisk standing will uncover the patients with insufficient QT adaptation abilities $[48,49]$. Both the QTc at maximal tachycardia (QT stretching) and the QTc after the heart rate has returned to baseline conditions (QT stunning) are of value. It should be noted that also controls will show QTc prolongation during these tests, so cut off values need to be increased. For QT stretching a QTc cut off value of $490 \mathrm{~ms}$ yielded a sensitivity of $89 \%$ and a sensitivity of 87\% for a Long QT diagnosis, knowing that patients with obviously prolonged supine QT intervals (i.e. $>480 \mathrm{~ms}$ for females, $470 \mathrm{~ms}$ for males) were already excluded [49]. Children, however, were not well represented in these stud- ies, which currently precludes the wide use of these cut off values in the pediatric population.

Another often-used technique is an exercise test. Especially the QTc at 4 minutes after maximal exercise discriminates between patients with Long QT syndrome and control patients [50]. In those patients who did not have an obviously prolonged QTc interval at baseline (i.e. $>480 \mathrm{~ms}$ for females, $470 \mathrm{~ms}$ for males), a 4-minute recovery QTc $\geq 445$ ms yielded in a sensitivity of $94 \%$ and a specificity of $90 \%$. Again, it should be noted that these values should be used with caution in a pediatric population [51]. Furthermore, both the brisk standing test as the exercise test, have not been meticulously studied in an unselected population suspected of Long QT syndrome. This precludes the solitary use of these tests at present.

And finally, when still in doubt, we certainly advice to discuss the case with a QT specialist. This is especially of importance when invasive therapeutic measurers are considered such as implantation of an ICD. Although beyond the scope of this paper, it should be noted that these patients, who are often young, can not be compared to the common ICD population with decreased left ventricular ejection fraction. These patients have an active life style with associated tachycardia and frequent maximal arm movements, which may complicate ICD therapy. Furthermore, there will be a lot of device replacements. Clearly, over-implantation of ICD's has occurred for many years in asymptomatic Long QT syndrome patients [52]. Also the decision for 1-lead, 2lead or subcutaneous ICD systems is critical and should be taken by an experienced team. This notwithstanding, the advice to avoid certain drugs with the ability to prolong the QT interval and result in arrhythmias, is of paramount importance and should be given to all Long QT patients [53].

\section{QT FUTURE}

There are many techniques already available to study QT adaptation in detail. However, these techniques are not yet ready for bedside use but some might be in the future. A lot of focus has been given to the value of variability of the QT interval that will be abnormal in patients with decreased repolarization reserve such as Long QT syndrome patients. As such, many different parameters have emerged to describe this variability. As variability in the QT interval is clearly a less static parameter than the measurement of a single QT of one beat during a supine ECG, it is hoped that this will provide a better measure to perform risk stratification and to monitor treatment effects. We here discuss only several of the many indices of QT variability.

For example, one of the earlier QT variability parameters is the 'QT variability index' (QTVI) that has been used to quantify the ratio between the variability in the QT interval and variability in heart rate. The equation QTVI = $\log _{10}\left[\left(\mathrm{QTV} / \mathrm{QTM}^{2}\right) /\left(\mathrm{HRV} / \mathrm{HRM}^{2}\right)\right]$ is derived from the variance in QT (QTV) in a certain time frame, normalized by its respective mean square $\left(\mathrm{QTM}^{2}\right)$, as a quotient of heart rate variability with the variance in heart rate (HRV) also normalized by its respective mean square $\left(\mathrm{HRM}^{2}\right)$. As this quotient is not normally distributed, the logarithm is taken to provide a normally distributed index $[54,55]$. Only few studies have actually found QTVI to be discriminative between 
Long QT syndrome patients and controls [55, 56]. As such, it has not attracted a lot of support yet in the search for discriminative QT indices in Long QT syndrome. This might be due to several limitations with this method, as for example described by Malik [57].

A related method to study QT variability is 'short term QT variability' (STVQT). In this method, beat-to-beat QT intervals are studied with the assumption that large between beat QT differences are more important (i.e. more arrhythmogenic) than a gradual increase or decrease in QT over several beats. Although the number of beats included is variable, an average of 30 beats is most common. The equation STVQT $=\Sigma \mid \mathrm{QT}_{\mathrm{n}+1}-\mathrm{QT}_{\mathrm{n}} / /(30 \times \sqrt{2})$ is derived from consecutive QT intervals ( $\mathrm{n}$ and $\mathrm{n}+1$ ) where STVQT is defined as the mean dispersion of these QT intervals perpendicular to the line-of-identity in a Poincaré plot [58]. The value of this parameter has only been studied by a few centers but seemed to be better suited to predict cardiac events in Long QT patients than a baseline QTc and to be better suited to predict Long QT mutation carriership [59, 60]. Again, also this method has drawbacks that should be acknowledged, and more studies are still underway.

A totally different method to study QT variability has recently been put forward by Mathias and colleagues [61]. In their study the standard deviation of QT among carriers of the same mutation was taken as a measure to study the risk of cardiac events. It appeared that in patients with type-1 Long QT syndrome (based on a mutation in the $K C N Q 1$ gene), every 20 ms increase in QTcSD (i.e. the QT standard deviation between carriers of the same $K C N Q 1$ gene mutation) resulted in a $33 \%$ increased risk for cardiac events after adjustment for the patient specific QTc. Although not an easy concept (the larger the variation between family members, the larger the risk for events), this parameter certainly awaits further study.

\section{QT CONCLUSION}

Although it is clear that an aberrant QT interval poses patients at risk for malignant arrhythmias and sudden death, the correct measurement and interpretation of the QT interval is not at all an easy task. We have discussed the history of the measurement of the QT interval, its current problems, and we have attempted to provide useful guidance on how to approach the measurement of the QT interval.

\section{CONFLICT OF INTEREST}

The authors confirm that this article content has no conflict of interest.

\section{ACKNOWLEDGEMENTS}

Declared none.

\section{REFERENCES}

[1] Taggart NW, Haglund CM, Tester DJ, Ackerman MJ. Diagnostic miscues in congenital long-QT syndrome. Circulation 2007; 115: 2613-20.

[2] Garg A, Lehmann MH. Prolonged QT interval diagnosis suppression by a widely used computerized ECG analysis system. Circ Arrhythm Electrophysiol 2013; 6: 76-83.
[3] Einthoven W. Ueber die Form des menschlichen Electrocardiogramms. Pflugers Arch Gesamte Physiol 1895; 60: 101-23.

[4] Einthoven W. Le telecardiogramme. Arch Int Physiol 1906; 4: 13264.

[5] Einthoven W. The different forms of the human electrocardiogram and their signification. Lancet 1912; 179: 853-61.

[6] Lewis T, Gilder MDD. The human electrocardiogram: a preliminary investigation of young male adults, to form a basis for pathological study. Philos Trans R Soc Lond B Biol Sci 1912; 202: 35176.

[7] Wolff L. Electrocardiography. Fundamentals and clinical application. Philadelphia: W.B. Saunders company 1950.

[8] Jervell A, Lange-Nielsen F. Congenital deaf-mutism, functional heart disease with prolongation of the Q-T interval and sudden death. Am Heart J 1957; 54: 59-68.

[9] Jervell A, Thingstad R, Endsjö TO. The surdo-cardiac syndrome: three new cases of congenital deafness with syncopal attacks and Q-T prolongation in the electrocardiogram. Am Heart J 1966; 72: 582-93.

[10] Postema PG, Van den Berg MP, Van Tintelen JP, et al. Founder mutations in the Netherlands. SCN5a 1795insD, the first described arrhythmia overlap syndrome and one of the largest and best described characterised families worldwide. Neth Heart J 2009; 17: 422-8.

[11] Romano C, Gemme G, Pongiglione R. Rare cardiac arrhythmias of the pediatric age. II. Syncopal attacks due to paroxysmal ventricular fibrillation [Italian]. Clin Pediatr (Bologna) 1963; 45: 656-683.

[12] Ward OC. A new familial cardiac syndrome in children. J Ir Med Assoc 1964; 54: 103-6.

[13] Keating M, Atkinson D, Dunn C, Timothy K, Vincent GM, Leppert M. Linkage of a cardiac arrhythmia, the long QT syndrome, and the Harvey ras-1 gene. Science 1991; 252: 704-6.

[14] Splawski I, Shen J, Timothy KW, et al. Spectrum of mutations in long-QT syndrome genes. KVLQT1, HERG, SCN5A, KCNE1, and KCNE2. Circulation 2000; 102: 1178-85.

[15] Gussak I, Brugada P, Brugada J, et al. Idiopathic short QT interval: a new clinical syndrome? Cardiology 2000; 94: 99-102.

[16] Villafañe J, Atallah J, Gollob MH, et al. Long-term follow-up of a pediatric cohort with short QT syndrome. J Am Coll Cardiol 2013; 61: 1183-91.

[17] Brugada R, Hong K, Dumaine R, et al. Sudden death associated with short-QT syndrome linked to mutations in HERG. Circulation 2004; 109: 30-5.

[18] Bellocq C, Van Ginneken AC, Bezzina CR, et al. Mutation in the KCNQ1 gene leading to the short QT-interval syndrome. Circulation 2004; 109: 2394-7.

[19] Priori SG, Pandit SV, Rivolta I, et al. A novel form of short QT syndrome (SQT3) is caused by a mutation in the KCNJ2 gene. Circ Res 2005; 96: 800-7.

[20] Woosley RL, Cossman J. Drug development and the FDA's Critical Path Initiative. Clin Pharmacol Ther 2007; 81: 129-33.

[21] Coronel R, De Bakker JM, Wilms-Schopman FJ, et al. Monophasic action potentials and activation recovery intervals as measures of ventricular action potential duration: experimental evidence to resolve some controversies. Heart Rhythm 2006; 3: 1043-50.

[22] Opthof T, Coronel R, Janse MJ. Is there a significant transmural gradient in repolarization time in the intact heart?: Repolarization gradients in the intact heart. Circ Arrhythm Electrophysiol 2009; 2: 89-96.

[23] Patel C, Burke JF, Patel H, et al. Is there a significant transmural gradient in repolarization time in the intact heart? Cellular basis of the $\mathrm{T}$ wave: a century of controversy. Circ Arrhythm Electrophysiol 2009; 2: 80-8.

[24] Janse MJ, Coronel R, Opthof T. Counterpoint: M cells do not have a functional role in the ventricular myocardium of the intact heart. Heart Rhythm 2011; 8: 934-7.

[25] Lepeschkin E, Surawicz B. The measurement of the Q-T interval of the electrocardiogram. Circulation 1952; 6: 378-88.

[26] Garson A. How to measure the QT interval--what is normal? Am J Cardiol 1993; 72: 14B-6B.

[27] Bazett HC. An analysis of the time-relations of electrocardiograms. Heart 1920; 7: 353-70.

[28] Moss AJ. Measurement of the QT interval and the risk associated with QTc interval prolongation: a review. Am J Cardiol 1993; 72: 23B-5B. 
[29] Moennig G, Schulze-Bahr E, Wedekind H, et al. Clinical value of electrocardiographic parameters in genotyped individuals with familial long QT syndrome. Pacing Clin. Electrophysiol 2001; 24: 406-15.

[30] Garson A Jr, Dick M 2nd, Fournier A, et al. The long QT syndrome in children. An international study of 287 patients. Circulation 1993; 87: 1866-72.

[31] Postema PG, Ritsema van Eck HJ, Opthof T, et al. IK1 modulates the U-wave: insights in a 100 year old enigma. Heart Rhythm 2009; 6: 393-400.

[32] Zhang L, Benson DW, Tristani-Firouzi M, et al. Electrocardiographic features in Andersen-Tawil syndrome patients with KCNJ2 mutations: characteristic T-U-wave patterns predict the KCNJ2 genotype. Circulation 2005; 111: 2720-6.

[33] Viskin S, Rosovski U, Sands AJ, et al. Inaccurate electrocardiographic interpretation of long QT: the majority of physicians cannot recognize a long QT when they see one. Heart Rhythm 2005; 2: 569-74.

[34] Postema PG, De Jong JS, Van der Bilt IA, Wilde AA. Accurate electrocardiographic assessment of the QT-interval: teach the tangent. Heart Rhythm 2008; 5: 1015-8.

[35] Goldenberg I, Moss AJ, Zareba W. QT interval: how to measure it and what is "normal". J Cardiovasc. Electrophysiol 2006; 17: 3336.

[36] Panicker GK, Karnad DR, Natekar M, Kothari S, Narula D, Lokhandwala Y. Intra- and interreader variability in QT interval measurement by tangent and threshold methods in a central electrocardiogram laboratory. J Electrocardiol 2009; 42: 348-52.

[37] Funck-Brentano C, Jaillon P. Rate-corrected QT interval: techniques and limitations. Am J Cardiol 1993; 72: 17B-22B.

[38] Fridericia LS. Die Systolendauer im Elektrokardiogramm bei normalen Menchen und bei Herzkranken. Acta Med Scand 1920; 53: 469-86.

[39] Luo S, Michler K, Johnston P, Macfarlane PW. A comparison of commonly used QT correction formulae: the effect of heart rate on the QTc of normal ECGs. J Electrocardiol 2004; 37 Suppl: 81-90.

[40] Barsheshet A, Peterson DR, Moss AJ, et al. Genotype-specific QT correction for heart rate and the risk of life-threatening cardiac events in adolescents with congenital long-QT syndrome. Heart Rhythm 2011; 8: 1207-13.

[41] Kirchhof P, Franz MR, Bardai A, Wilde AM. Giant T-U waves precede Torsades de Pointes in Long QT syndrome. A systematic electrocardiographic analysis in patients with acquired and congenital QT prolongation. J Am Coll Cardiol 2009; 54: 143-9.

[42] Viskin S. The QT interval: too long, too short or just right. Heart Rhythm 2009; 6: 711-5.

[43] Crotti L, Monti MC, Insolia R, et al. NOS1AP is a genetic modifier of the Long-QT syndrome. Circulation 2009; 120: 1657-63.

[44] Goldenberg I, Horr S, Moss AJ, et al. Risk for life-threatening cardiac events in patients with genotype-confirmed Long-QT syndrome and normal-range corrected QT intervals. J Am Coll Cardiol 2010; 57: 51-9.

[45] Hofman N, Wilde AA, Kaab S, et al. Diagnostic criteria for congenital long QT syndrome in the era of molecular genetics: do we need a scoring system? Eur Heart J 2007; 28: 575-80.

[46] Priori SG, Schwartz PJ, Napolitano C, et al. Risk stratification in the long-QT syndrome. N Engl J Med 2003; 348: 1866-74.
[47] Tomás M, Napolitano C, De Giuli L, et al. Polymorphisms in the NOS1AP gene modulate QT interval duration and risk of arrhythmias in the long QT syndrome. J Am Coll Cardiol 2010; 55: 274552.

[48] Viskin S, Postema PG, Bhuiyan ZA, et al. The response of the QTinterval to the brief tachycardia provoked by standing. A bedside test for diagnosing Long-QT syndrome. J Am Coll Cardiol 2010; 55: 1955-61.

[49] Adler A, Van der Werf C, Postema PG, et al. The phenomenon of "QT stunning": The abnormal QT prolongation provoked by standing persists even as the heart rate returns to normal in patients with long QT syndrome. Heart Rhythm 2012; 9: 901-8.

[50] Sy RW, Van der Werf C, Chattha IS, et al. Derivation and validation of a simple exercise-based algorithm for prediction of genetic testing in relatives of LQTS probands. Circulation 2011; 124: 2187-94.

[51] Berger WR, Gow RM, Kamberi S, Cheung M, Smith KR, Davis AM. The QT and corrected QT interval in recovery after exercise in children. Circ Arrhythm Electrophysiol 2011; 4: 448-55.

[52] Schwartz PJ, Spazzolini C, Priori SG, et al. Who are the Long-QT syndrome patients who receive an implantable cardioverterdefibrillator and what happens to them? Data from the European Long-QT syndrome implantable cardioverter-defibrillator (LQTS ICD) registry. Circulation 2010; 122: 1272-82.

[53] Postema PG, Neville J, De Jong JS, Romero K, Wilde AA, Woosley RL. Safe drug use in Long QT syndrome and Brugada syndrome: www.QTdrugs.org and www.BrugadaDrugs.org. Europace 2013; 15: 1042-9.

[54] Berger RD, Kasper EK, Baughman KL, Marban E, Calkins H, Tomaselli GF. Beat-to-beat QT interval variability: novel evidence for repolarization lability in ischemic and nonischemic dilated cardiomyopathy. Circulation 1997; 96: 1557-65.

[55] Bilchick K, Viitasalo M, Oikarinen L, Fetics B, Tomaselli G, Swan H, Laitinen PJ, Vaananen H, Kontula K, Berger RD. Temporal repolarization lability differences among genotyped patients with the long QT syndrome. Am J Cardiol 2004; 94: 1312-6.

[56] Nemec J, Buncova M, Shusterman V, Winter B, Shen WK, Ackerman MJ. QT interval variability and adaptation to heart rate changes in patients with long QT syndrome. Pacing Clin Electrophysiol 2009; 32: 72-81.

[57] Malik M. Beat-to-beat QT variability and cardiac autonomic regulation. Am. J. Physiol. Heart Circ Physiol 2008; 295: H923-5.

[58] Thomsen MB, Verduyn SC, Stengl M, et al. Increased short-term variability of repolarization predicts d-sotalol-induced torsades de pointes in dogs. Circulation 2004; 110: 2453-9.

[59] Hinterseer M, Thomsen MB, Beckmann BM, et al. Beat-to-beat variability of QT intervals is increased in patients with druginduced long-QT syndrome: a case control pilot study. Eur Heart J 2008; 29: 185-90.

[60] Hinterseer M, Beckmann BM, Thomsen MB, et al. Relation of increased short-term variability of QT interval to congenital longQT syndrome. Am J Cardiol 2009; 103: 1244-8.

[61] Mathias A, Moss AJ, Lopes CM, et al. Prognostic implications of mutation specific QTc standard deviation in congenital Long QT syndrome. Heart Rhythm 2013; 10: 720-5. 